\title{
Delayed Occurrence of Escherichia coli Subdural Empyema Following Head Injury in an Elderly Patient: A Case Report and Literature Review
}

\author{
Thangaraj Munusamy ${ }^{1}$ Shree Kumar Dinesh ${ }^{1}$ \\ ${ }^{1}$ Department of Neurosurgery, National Neuroscience Institute, \\ Singapore \\ J Neurol Surg Rep 2015;76:e79-e82.
}

\begin{abstract}
Address for correspondence Thangaraj Munusamy, MA, MB, BChir, Department of Neurosurgery, National Neuroscience Institute, 11, Jalan Tan Tock Seng, Singapore 308433, Singapore

(e-mail: munusamy@cantab.net).
\end{abstract}

\begin{abstract}
Keywords

- subdural empyema

- head injury

- antibiotic therapy

- chronic subdural hematoma

Subdural empyema is a rare but serious intracranial infection that warrants prompt management to reduce morbidity and avoid mortality. However, clinical and radiologic features may be subtle or ambivalent. Thus a diagnosis of subdural empyema should not be discounted, especially in a patient with a history of head trauma. Treatment consists of surgery to establish bacteriologic identification and subsequently guide antibiotic therapy. Here we present a case of delayed Escherichia coli subdural empyema following a head injury in an elderly patient without significant risk factors. Computed tomography imaging was equivocal for subdural empyema. The patient underwent surgery and was treated with intravenous antibiotic therapy. Although initial improvement in the patient's clinical condition was observed, he eventually succumbed to nosocomial pneumonia. In this article, we discuss the presentation, diagnostic tools, and treatment options for subdural empyema with an emphasis on the challenges. The management conundrum that follows prompted us subsequently to review the literature.
\end{abstract}

\section{Introduction}

Subdural empyema is a rare intracranial infection associated with high morbidity and mortality. ${ }^{1,2}$ Outcome depends on patient age, general condition and comorbidities, causative organism, primary source, and prompt diagnosis and treatment. ${ }^{3}$ Cases of subdural empyema following head trauma or surgical procedures in adults have been reported; however, very few were caused by Escherichia coli. ${ }^{4-6}$ Furthermore, a delayed occurrence of $E$. coli subdural empyema following head injury is rarely reported. We present a patient who was treated with craniectomy and pus evacuation followed by intravenous antibiotic therapy.

\section{Case Report}

A 77-year-old man with a history of degenerative spine disease sustained a 2-mm thin right temporoparietal acute subdural hematoma (SDH) following a fall. There were no associated skull fractures or cerebrospinal fluid leakage. He was managed conservatively and discharged. One month later, he was readmitted with headache and dysphasia. On admission, he was afebrile with a Glasgow Coma Score (GCS) of 15. Physical examination was notable only for mild expressive dysphasia. Laboratory investigations revealed raised peripheral blood leukocytes with polymorphonuclear predominance. Plain computed tomography (CT) imaging of the head revealed a subacute to chronic SDH measuring $1.8 \mathrm{~cm}$ thick, overlying the left frontoparietal convexity, causing mass effect and subtle contralateral shift of midline structures ( $\mathbf{- F i g}$. 1A). The previously noted thin right temporoparietal SDH had resolved, and the visualized paranasal sinuses and mastoid air cells were well pneumatized. As in the previous scan, no skull fractures were detected.

The patient was prepared for burr hole surgery; however, he spiked a temperature of $39^{\circ} \mathrm{C}$. In view of the fever, a full septic investigation was performed, and the decision was made received

June 28, 2014

accepted

December 15, 2014

published online

March 23, 2015
DOI http://dx.doi.org/

10.1055/s-0035-1547366. ISSN 2193-6366. (c) 2015 Georg Thieme Verlag KG
Stuttgart · New York

License terms

(1) $\circledast$ 

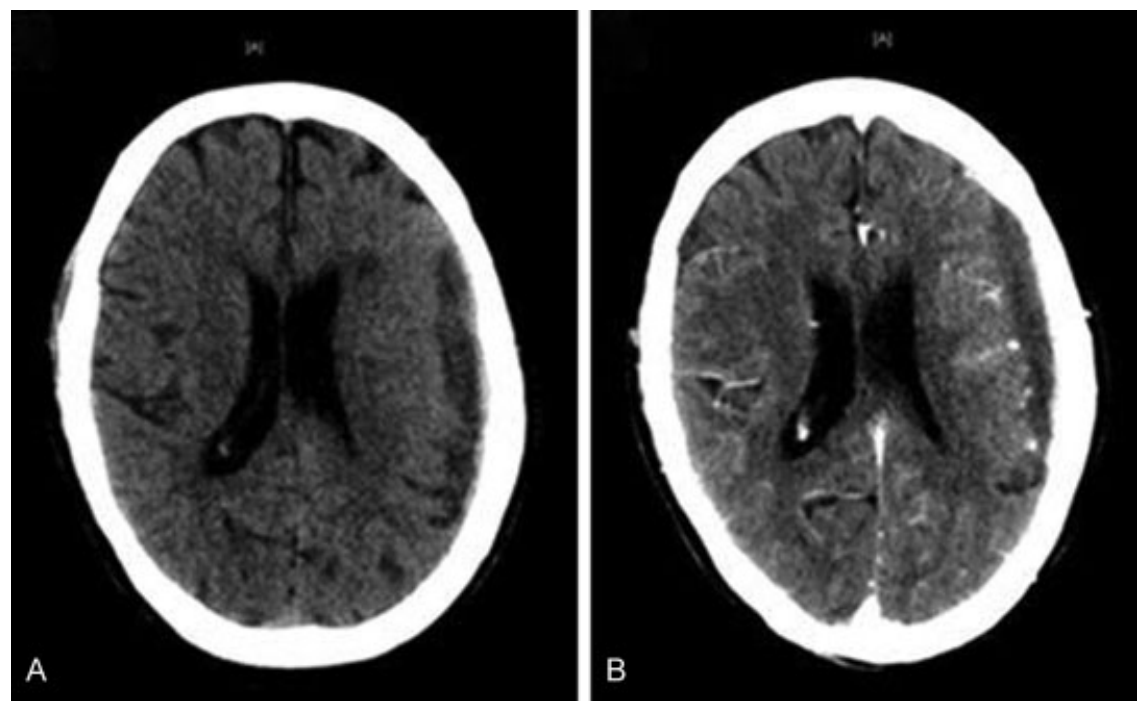

Fig. 1 (A) Computed tomography (CT) scan of the head showing a $1.8 \mathrm{~cm}$ left frontoparietal convexity subdural collection with mass effect and subtle midline shift. (B) Contrast-enhanced CT scan of the head showing a stable left frontoparietal convexity subdural collection with no overt features of enhancement.

to postpone surgery and start empirical intravenous co-amoxiclav. Serum investigation revealed elevated C-reactive protein and procalcitonin levels. Urine and blood cultures were negative, and chest radiograph did not show any lung abnormality. Despite intravenous co-amoxiclav, the patient continued to be febrile and became increasingly confused and lethargic with GCS E3V4M6. Repeat blood investigations revealed an increasing trend of inflammatory markers. Subsequently the patient had a drop in limb power on the right side. A contrast-enhanced CT of the head and sinuses was obtained that showed stable left frontoparietal convexity SDH without abnormal enhancement (-Fig. 1B). Nevertheless, antibiotic therapy was converted to piperacillin and tazobactam. In view of potential functional compromise, the decision was made to proceed with surgical evacuation of the left SDH. Because the contrast CT was not conclusive for subdural empyema, the preoperative diagnosis was left as chronic SDH and the patient was prepared for the burr hole procedure.

A double burr hole trephination was performed that revealed thick greenish purulent material. Because pus was refractory to Ringer lactate irrigation, surgery was converted to a craniectomy. Specimens were sent for microbiological investigation. The brain was noted to be swollen after pus evacuation; thus the bone flap was left out and an intracranial pressure (ICP) monitor probe was inserted. Postoperatively the patient was kept intubated in the intensive care unit. An urgent infectious disease consult was made, and antibiotic therapy was escalated to meropenem and vancomycin. ICP remained within normal limits, and the patient was extubated on postoperative day 2. Postextubation, GCS was E4V2M6 and his right side remained weak.

Culture of the purulent specimen identified Escherichia coli as the causative organism. Because the $E$. coli strain showed sensitivity to most antibiotics, antibiotic therapy was deescalated to intravenous ceftriaxone. Initial improvement in fever and inflammatory markers was seen, but unfortunately the patient deteriorated and succumbed to nosocomial pneumonia on postoperative day 19 .

\section{Discussion}

Subdural empyema is a neurosurgical emergency associated with high mortality. ${ }^{1,2}$ Infection of the paranasal or mastoid sinuses and otitis media are the most common causes. ${ }^{7,8}$ Other causes include direct spread from meningitis, hematogenous spread from distant sites, and postcranial surgery or head trauma, particularly in cases where there is a compound depressed fracture. ${ }^{2,9}$ Spontaneous subdural empyema is extremely rare, and the few cases reported were associated with minor head trauma. ${ }^{10}$ Interestingly, delayed presentations of subdural empyema were reported to occur even 20 years after head trauma. ${ }^{11}$ This could well be a result of secondary infection of a chronic subdural hematoma or hygroma. ${ }^{4,5,12}$

The causative organisms varied according to the primary source of infection. In subdural empyema secondary to mastoiditis or paranasal sinusitis, aerobic and microaerophilic streptococci are the usual culprits. ${ }^{13,14}$ In infants and young children, subdural empyema was commonly caused by direct spread from Haemophilus influenzae and Streptococcus pneumoniae meningitis. ${ }^{15}$ Staphylococcus aureus was seen in postoperative and posttraumatic subdural empyema, whereas in the immunocompromised it is not uncommon to isolate Klebsiella pneumonia. ${ }^{7}$ Other causative organisms for subdural empyema that have been reported were E. coli and Salmonella species. ${ }^{4,6,9,12}$ In general, the success rate for culturing bacteria from surgically evacuated subdural pus ranged between $54 \%$ and $81 \%^{16-18}$ The use of broad-spectrum antibiotics for treating meningitis and sinusitis could explain the low culture positivity in some studies. ${ }^{14,18}$ In our case, despite the use of intravenous broad-spectrum antibiotics in the first 2 days of admission, pus culture grew significant $E$. coli.

Infants and the elderly are at the highest risk for $E$. coli infection. ${ }^{19}$ Hematogenous spread of $E$. coli is usually a complication of urinary or gastrointestinal tract infection; however, occasionally no reliable primary source is 
identified. ${ }^{20}$ The limited understanding of $E$. coli infection is apparent in bacterial meningitis in infants where the predisposing factors were not identified in $70 \%$ of patients. ${ }^{21}$ In our case, no extracranial infective focus was identified.

On plain CT imaging, subdural empyema typically resembles subdural hematoma in their crescent shape and relationship to sutures. With contrast media, an enhancing rim is typically identified. ${ }^{20}$ However, contrast-enhanced CT may appear normal or show subtle characteristics. ${ }^{22}$ Furthermore, it has been reported that in chronic cases, a well-organized abscess may fail to generate peripheral enhancement. ${ }^{23}$ We encountered this in our case where contrast-enhanced CT head did not manifest any significant enhancement, and we noted that the evacuated subdural pus was viscid and organized. In retrospect, it may have been valuable to perform magnetic resonance (MR) imaging because it has been shown to be superior to $\mathrm{CT}$ in demonstrating fluid collection with enhancement. ${ }^{20}$ In fact, diffusion-weighted MR imaging may reliably distinguish subdural empyema from subdural hematoma or reactive subdural effusion that may also occur after trauma, surgery, or meningitis. ${ }^{24}$

The mainstay of treatment for subdural empyema consists of surgical evacuation of pus, eradication of the primary foci of infection, and adequate antibiotic therapy. ${ }^{9,14,16}$ Local administration of antibiotics is usually not necessary, and occasionally medical management alone may suffice. ${ }^{25,26}$ Surgical procedures vary among burr hole, craniotomy, and craniectomy, and controversy exists over the surgical procedure of choice. ${ }^{27}$ Many authors have shown that results of all three approaches are comparable. ${ }^{13,14,28}$ Burr hole drainage alone has been shown to be sufficient for the complete drainage of subdural empyema. ${ }^{13,21}$ However, some found that the success rate with craniotomy was superior over burr holes and associated with a lower mortality rate. ${ }^{8,17}$ Furthermore, in one study burr hole evacuation of pus resulted in a $40 \%$ recurrence. $^{29}$

In general, if the pus consistency is thin and there are no loculated collections, evacuation via burr holes is adequate and could avoid potential surgical complications. However, if pus is thick or well organized, a craniotomy is recommended. ${ }^{14}$ In some cases, the underlying brain may be swollen; hence bone flap must be left out. ${ }^{20,30}$ Interestingly, one study reported that in patients with no or a slight impairment of consciousness, craniotomy might be preferred, whereas in stuporous or comatose patients, burr holes were advised instead. ${ }^{16}$ In our case, a craniectomy was performed that provided a direct route for sufficient pus evacuation and allowed expansion of the swollen brain without any compromise in ICP.

\section{Conclusions}

Subdural empyema should be suspected in posttraumatic elderly patients with unexplained fever and abnormal CT imaging suggestive of a subdural collection. MR imaging may provide an additional advantage if contrast-enhanced CT imaging is equivocal for empyema. Treatment of subdural empyemas comprises early surgical intervention, identification of the causative organism, and administration of appropriate antibiotic agents.

\section{References}

1 Le Beau J, Creissard P, Harispe L, Redondo A. Surgical treatment of brain abscess and subdural empyema. J Neurosurg 1973;38(2): 198-203

2 Post EM, Modesti LM. "Subacute" postoperative subdural empyema. J Neurosurg 1981;55(5):761-765

3 Van Alphen HA, Dreissen JJ. Brain abscess and subdural empyema. Factors influencing mortality and results of various surgical techniques. J Neurol Neurosurg Psychiatry 1976;39(5): 481-490

4 Bakker S, Kluytmans J, den Hollander JC, Lie ST. Subdural empyema caused by Escherichia coli: hematogenous dissemination to a preexisting chronic subdural hematoma. Clin Infect Dis 1995; 21(2):458-459

5 Miedema CJ, Kimpen JL. Hematogenous dissemination of Escherichia coli to a preexistent subdural hematoma in a child. Clin Infect Dis 1996;23(3):662

6 Vetrovec GW, Warner JF. Infected subdural hematoma: three case reports involving gram-negative organisms. Am J Med Sci 1975; 269(1):113-115

7 Greenlee JE. Subdural empyema. Curr Treat Options Neurol 2003; $5(1): 13-22$

8 Yilmaz N, Kiymaz N, Yilmaz C, et al. Surgical treatment outcome of subdural empyema: a clinical study. Pediatr Neurosurg 2006; 42(5):293-298

9 Dill SR, Cobbs CG, McDonald CK. Subdural empyema: analysis of 32 cases and review. Clin Infect Dis 1995;20(2):372-386

10 Bachmeyer C, Logak M, Ammouri W, Blanc A-S. Spontaneous Escherichia coli meningitis with subdural empyema in an adult. South Med J 2005;98(12):1225-1226

11 Tokoro K, Yamataki A, Nakajima F. Subdural empyema occurring 20 years after trauma: case report. Neurosurgery 1987;21(5): 724-726

12 Casson IR, Patel P, Blair D, Bergtraum M. Subdural empyema; caused by infection of preexisting subdural hematoma. N Y State J Med 1981;81(3):389-391

13 Bok AP, Peter JC. Subdural empyema: burr holes or craniotomy? A retrospective computerized tomography-era analysis of treatment in 90 cases. J Neurosurg 1993;78(4):574-578

14 Miller ES, Dias PS, Uttley D. Management of subdural empyema: a series of 24 cases. J Neurol Neurosurg Psychiatry 1987;50(11): 1415-1418

15 Wu T-J, Chiu N-C, Huang F-Y. Subdural empyema in children-20year experience in a medical center. J Microbiol Immunol Infect 2008;41(1):62-67

16 Mauser HW, Van Houwelingen HC, Tulleken CA. Factors affecting the outcome in subdural empyema. J Neurol Neurosurg Psychiatry 1987;50(9):1136-1141

17 Nathoo N, Nadvi SS, van Dellen JR, Gouws E. Intracranial subdural empyemas in the era of computed tomography: a review of 699 cases. Neurosurgery 1999;44(3):529-535; discussion 535-536

18 Tewari MK, Sharma RR, Shiv VK, Lad SD. Spectrum of intracranial subdural empyemas in a series of 45 patients: current surgical options and outcome. Neurol India 2004;52(3):346-349

19 Laupland KB, Gregson DB, Church DL, Ross T, Pitout JDD. Incidence, risk factors and outcomes of Escherichia coli bloodstream infections in a large Canadian region. Clin Microbiol Infect 2008; 14(11): 1041-1047

20 Rich PM, Deasy NP, Jarosz JM. Intracranial dural empyema. Br J Radiol 2000;73(876):1329-1336 
21 Iimura Y, Tsutsumi S, Mitome Y, et al. Infantile chronic subdura hematoma infected by Escherichia coli-case report. Neurol Med Chir (Tokyo) 2010;50(6):482-484

22 Moseley IF, Kendall BE. Radiology of intracranial empyemas, with special reference to computed tomography. Neuroradiology 1984 26(5):333-345

23 Smirniotopoulos JG, Murphy FM, Rushing EJ, Rees JH, Schroeder JW. Patterns of contrast enhancement in the brain and meninges. Radiographics 2007;27(2):525-551

24 Chen CY, Huang CC, Chang YC, Chow NH, Chio CC, Zimmerman RA. Subdural empyema in 10 infants: US characteristics and clinical correlates. Radiology 1998;207(3):609-617

25 Leys D, Destee A, Petit H, Warot P. Management of subdura intracranial empyemas should not always require surgery. J Neurol Neurosurg Psychiatry 1986;49(6):635-639
26 Mauser HW, Ravijst RA, Elderson A, van Gijn J, Tulleken CA. Nonsurgical treatment of subdural empyema. Case report. J Neurosurg 1985;63(1):128-130

27 Pathak A, Sharma BS, Mathuriya SN, Khosla VK, Khandelwal N, Kak VK. Controversies in the management of subdural empyema. A study of 41 cases with review of literature. Acta Neurochir (Wien) 1990;102(1-2):25-32

28 Shearman CP, Lees PD, Taylor JC. Subdural empyema: a rational management plan. The case against craniotomy. Br J Neurosurg 1987;1(2):179-183

29 Feuerman T, Wackym PA, Gade GF, Dubrow T. Craniotomy improves outcome in subdural empyema. Surg Neurol 1989;32(2):105-110

30 Ong YK, Goh KYC, Chan C. Bifrontal decompressive craniectomy for acute subdural empyema. Childs Nerv Syst 2002;18(6-7): 340-343; discussion 344 